\title{
Polypharmacy and adverse drug events among propensity score matched privately insured persons with and without spinal cord injury
}

\author{
Brittany N. Hand ${ }^{1} \cdot$ James S. Krause ${ }^{1} \cdot$ Kit N. Simpson ${ }^{1}$
}

Received: 15 August 2017 / Revised: 11 December 2017 / Accepted: 12 December 2017 / Published online: 23 January 2018

(c) International Spinal Cord Society 2018

\begin{abstract}
Study design Retrospective quasi-experimental design.

Objectives To compare the incidence of adverse drug events (ADEs) between persons with and without spinal cord injury (SCI), while controlling for all potential and available risk factors.

Setting A commercially available claims dataset consisting of $\sim 170$ million patient cases in the United States between 2012 and 2013.

Methods Participants (aged 18-64 years) included 2779 persons with polypharmacy and traumatic or non-traumatic SCI and 2779 propensity score-matched persons with polypharmacy without SCI. The cohorts were matched using demographic variables including number of concomitant prescriptions, comorbidities, hospital admissions, age, gender, and geographic region. Inpatient and outpatient claims records containing 395 distinct IDC-9 codes indicative of ADEs were extracted. Incidence and frequency of ADEs were compared between groups using logistic and Poisson regression, respectively.

Results Persons with SCI were significantly more likely to experience an ADE than matched controls (Odds Ratio $=1.45$, $p<0.0001)$. Among persons with ADEs $(n=1552)$, individuals with SCI experienced fewer ADEs over time than matched controls (Incidence Rate Ratio $=0.91, p<0.0001$ ).

Conclusions While persons with SCI and polypharmacy are at a greater risk for experiencing an ADE, their medical care after an ADE may be better managed than that of a matched control population. There may be a need for practice guidelines that facilitate proactive identification of persons with SCI at the highest risk of ADE. Steps may then be taken to mitigate risk, in contrast to current practice trends that appear to take a reactive approach after an ADE has occurred.
\end{abstract}

\section{Introduction}

Polypharmacy is commonly defined as the concomitant use of five or more prescription drugs [1]. Numerous negative health consequences are associated with polypharmacy, including increased risk of falls, adverse drug events (ADEs), hospitalization, mortality, declines in functional status, and impaired cognition [2]. Evidence suggests that rate of polypharmacy is increasing and is associated with individuals' risk for serious drug-drug interactions [1]. A number of individual characteristics are associated with increased risk of polypharmacy including older age [1],

James S. Krause

krause@musc.edu

1 College of Health Professions, Medical University of South Carolina, Charleston, USA geographic region of residence [3], and medical comorbidities [4]. As a result, there is a widespread effort to increase awareness of the dangers of polypharmacy and to ensure that the benefits and risks are adequately balanced when prescribing multiple medications $[5,6]$.

Prescription of multiple medications is common in treating patients with spinal cord injury (SCI). Research suggests that, on average, there is a greater than three-fold increase in the number of medications taken by an individual after sustaining a SCI [7]. This increase in medications can be problematic, however, as higher numbers of prescription medications are associated with depression [8], fatigue [9], and increased risk of pressure ulcers [10] in persons with SCI. Until recently, relatively little was known about the incidence of polypharmacy in this population. In 1998, Hope and Kailis [11] reported an average of more than eight prescription medications among 88 participants with SCI who were treated at a regional SCI center. More 


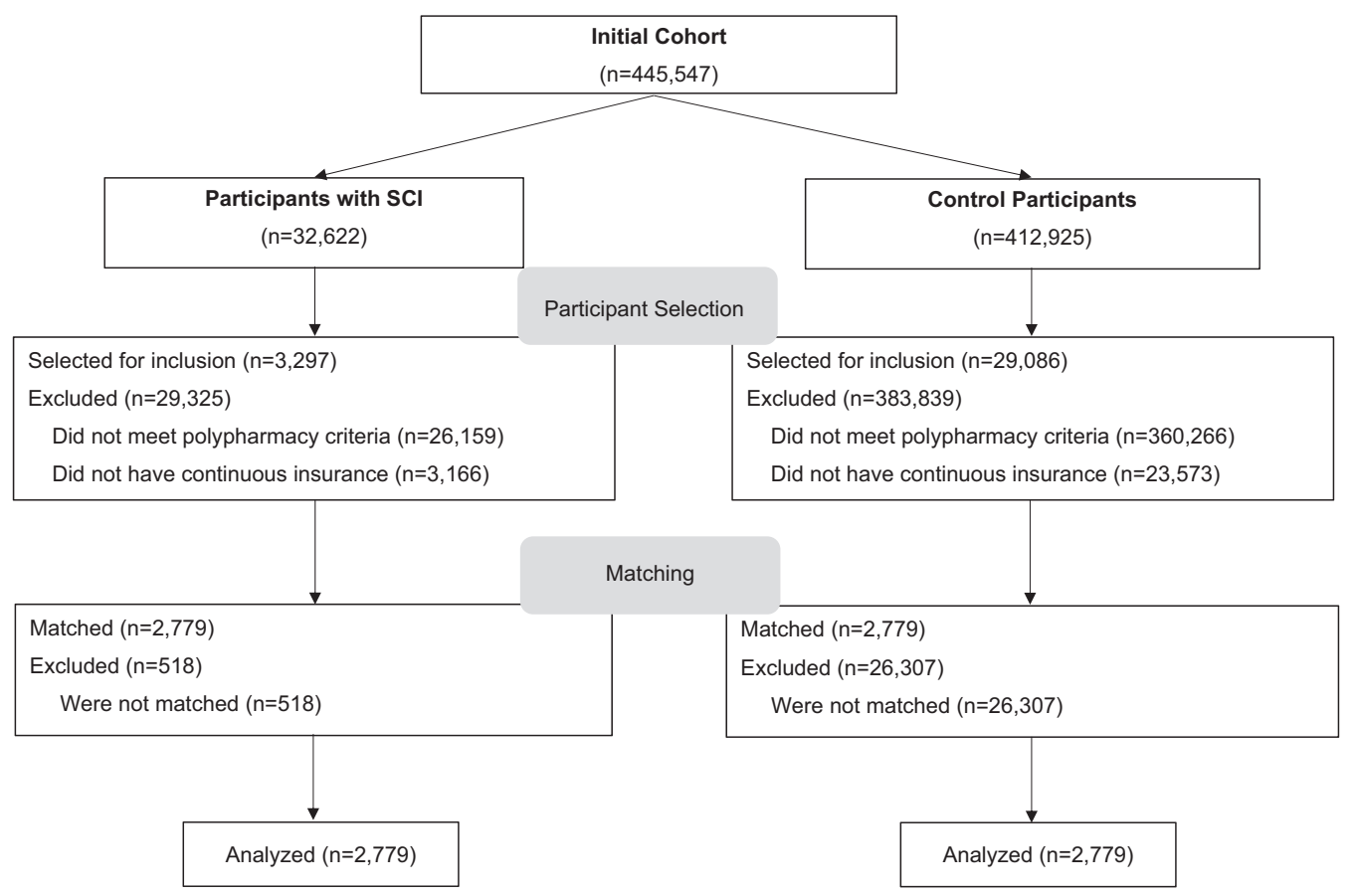

Fig. 1 Flow diagram representing the selection and matching process for study participants

recent work [12] suggests that $30 \%$ of adults with pediatric onset SCI and $56 \%$ of persons with adult onset traumatic or non-traumatic SCI [13] take five or more concomitant medications. Among adults with pediatric onset SCI, those with tetraplegia and more secondary health conditions have the highest rates of polypharmacy [12]. Moreover, when compared to an age and gender-matched control population, persons with SCI are prescribed with significantly more total medications, drugs from high-risk categories, and multiple medications within drug categories [13]. These prescription medication patterns place persons with SCI at significantly greater risk for ADEs than persons without SCI [13].

The aforementioned studies examining polypharmacy in SCI provided valuable information about the incidence of this phenomenon. Specifically, these findings revealed that differences in the number of prescription medications [13] and medical comorbidities [12] are important, potentially confounding variables that should be considered when comparing risk for ADE between persons with and without SCI. Findings from studies of other diagnostic populations suggest that hospitalizations [14], age [1], gender [15], and geographic region of residence [3] are also important characteristics to consider. The purpose of the present study is to build upon previous literature by comparing the incidence of ADEs between persons with and without SCI, while controlling for all available risk factors. Based on literature to date, we hypothesize that after controlling for all available risk factors, persons with SCI will demonstrate greater incidence and frequency of ADEs than matched controls.

\section{Methods}

\section{Data source}

Data used for the analysis were derived from Thompson Reuters MarketScan ${ }^{\circledR}$ Commercial Claims and Encounters Databases for the years 2012-2013. These databases represent $\sim 170$ million employees, dependents, and retirees. De-identified individual-level healthcare claims data for inpatient, outpatient, and prescription drug services were used in this analysis.

\section{Participant selection and study period}

From the available data, cohorts of persons with and without SCI were formed (Fig. 1). Using the same SCI phenotype as Kitzman et al. [13], initial identification of persons aged 18-64 years with SCI was based on an outpatient medical claim during the year 2012 with an SCI diagnostic code of 344.0x (quadriplegia and quadriparesis), 344.1x (paraplegia), 806.xx (fracture of vertebral column with SCI), or 952.xx (SCI without evidence of spinal bone injury). As a result of using these diagnostic codes, the SCI cohort included persons with traumatic (ICD-9 806, 952) or non-traumatic SCI (ICD-9 344). The first six months following the first date of service in 2012 represented the baseline period. The number of concomitant medications per month during the baseline period was extracted from individual-level prescription drug records. As the objective 
of the study was to characterize ADE patterns associated with polypharmacy, participants were required to have five or more concomitant medications in at least four of the six baseline months. Participants were also required to have at least 12 months of continuous insurance coverage after the date of the baseline period.

Participants included in the control cohort met the same eligibility criteria as the SCI participants, but did not have a diagnosis of SCI. Propensity score matching was conducted using a logistic regression model to predict the probability that, given a set of observed covariates, a participant has a SCI [16]. This model resulted in a propensity score derived from the following baseline characteristics: number of concomitant prescriptions per month during each month of the baseline period, age, sex, health plan type, geographical region, hospital admissions during baseline (yes/ no), number of emergency room visits, number of high-risk prescriptions, and number of days' supply of high-risk prescriptions during baseline. Drug classes considered high risk were: (1) sedative-hypnotic, non-barbiturates, (2) anxiolytics, (3) antidepressants, (4) analgesic-narcotics, (5) anticonvulsants, and (6) skeletal muscle relaxants [13]. Additionally, scores on two comorbidity indices-the Charlson Comorbidity Index [17] and Elixhauser Index [18] were used in propensity score matching. The Elixhauser Index includes 31 dichotomous variables indicating the presence or absence of comorbid diagnoses including, but not limited to: alcohol abuse, drug abuse, obesity, diabetes, and psychological conditions (e.g., depression, anxiety, or psychosis). Including such variables in propensity score matching ensures similarity between the two cohorts, so that observed differences in outcomes can be more confidently ascribed to the distinguishing factor (SCI diagnosis). Matching was performed without replacement using a greedy nearest available neighbor algorithm [19]. The quality of the match was confirmed with standardized mean differences on observed variables $<0.25$ and graphical displays of propensity score distributions in the unmatched and matched cohorts [20].

\section{Measures}

Demographic information was captured during the baseline period for both cohorts. Information about the number of ADEs was extracted from inpatient and outpatient medical claims during the 12-month post-baseline period. Indicators of ADEs consisted of 395 diagnostic codes identified by the Expert Panel for Classification of Adverse Event ICD-9-CM Codes [21]. Outcome variables included a dichotomous indicator of whether or not a participant had any ADE ICD-9 code and a count variable of the frequency of ADEs per participant during the post-baseline period.

\section{Analyses}

Participant demographics were summarized descriptively. Conditional logistic regression, to account for matched pairs, was used to compare differences between cohorts on the risk of having any ADE during the post-baseline period, while controlling for the following variables: gender, comorbidities, age in decades, average number of prescriptions per month during baseline, and whether or not the participant had a hospitalization during baseline. Among participants with at least one ADE, differences between cohorts in the number of ADEs during the post-baseline period were examined using Poisson regression with a random effect for the matched pairs and a log link. Incidence rate ratios (IRR), which represent the ADE incidence rate in persons with SCI divided by the ADE incidence rate in persons without SCI, 95\% confidence intervals of the IRR, and two-sided $P$ values for testing the hypothesis that the IRR is 1.0 were examined. Analyses were conducted using SAS software version 9.4 [22].

\section{Statement of ethics}

We certify that all applicable institutional and governmental regulations concerning the ethical use of human volunteers were followed during the course of this research.

\section{Results}

The SCI and control cohorts each consisted of 2779 participants who met all eligibility criteria. Demographic information about participants is shown in Table 1. Both cohorts were slightly over $50 \%$ female. The average age of participants in the SCI cohort was 50.1 (standard deviation [SD] $=10.6)$, and average age of control participants was 48.8 ( $\mathrm{SD}=11.8)$. Both cohorts were comprised of predominantly individuals residing in the Southern United States $(\mathrm{SCI}=37.1 \%$, Controls $=41.1 \%)$ with Preferred Provider Organization (PPO) insurance plans (SCI $=61.1 \%$, Controls $=62.2 \%$ ). Additionally, both cohorts were characterized by a low comorbidity burden at baseline, as indicated by the mean values for the comorbidity indices. The standardized mean differences of quantitative variables included in the propensity score model before and after matching are shown in Fig. 2. As all variables had standardized mean differences $<0.25$, the cohorts were deemed to be well-matched and suitable for subsequent analyses.

Level-specific SCI diagnostic codes were identified for $32.7 \%$ of participants in the SCI cohort. Among those with 
Table 1 Participant demographic characteristics during baseline

\begin{tabular}{|c|c|c|}
\hline & $\begin{array}{l}\text { SCI \% } \\
(n=2779)\end{array}$ & $\begin{array}{l}\text { Controls \% } \\
(n=2779)\end{array}$ \\
\hline \multicolumn{3}{|l|}{ Gender } \\
\hline Female & 52.3 & 53.1 \\
\hline Male & 47.7 & 46.9 \\
\hline $\operatorname{Age}^{a}$ & $48.8 \pm 11.8$ & $50.1 \pm 10.6$ \\
\hline \multicolumn{3}{|l|}{ US region of residence } \\
\hline South & 37.1 & 41.1 \\
\hline North central & 25.3 & 22.5 \\
\hline West & 23.5 & 20.9 \\
\hline Northeast & 12.9 & 13.9 \\
\hline Unknown & 1.3 & 1.8 \\
\hline \multicolumn{3}{|l|}{ Insurance plan } \\
\hline PPO & 61.0 & 62.2 \\
\hline HMO & 15.4 & 14.0 \\
\hline Non-capitated POS & 7.8 & 9.0 \\
\hline Comprehensive & 6.8 & 3.5 \\
\hline Consumer-driven health plan & 5.2 & 6.6 \\
\hline High deductible health plan & 2.2 & 3.1 \\
\hline $\mathrm{EPO}$ & 1.0 & 1.0 \\
\hline POS & 0.6 & 0.6 \\
\hline \multicolumn{3}{|l|}{ SCI Level } \\
\hline $\mathrm{C} 1-\mathrm{C} 4$ & 5.1 & - \\
\hline $\mathrm{C} 5-\mathrm{C} 7$ & 2.4 & - \\
\hline T1-T6 & 1.4 & - \\
\hline $\mathrm{T} 7-\mathrm{T} 12$ & 7.8 & - \\
\hline Lumbar/sacral & 12.5 & - \\
\hline Unknown & 67.3 & - \\
\hline \multicolumn{3}{|l|}{ SCI mechanism ${ }^{\mathrm{b}}$} \\
\hline Non-traumatic & 63.3 & - \\
\hline Traumatic & 36.7 & - \\
\hline $\begin{array}{l}\text { Emergency room visit during } \\
\text { baseline }\end{array}$ & 29.7 & 27.0 \\
\hline Hospitalization during baseline & 25.0 & 17.1 \\
\hline Charlson index & $0.21 \pm 0.9$ & $0.22 \pm 0.9$ \\
\hline $\begin{array}{l}\text { Baseline concomitant } \\
\text { medications }\end{array}$ & $8.8 \pm 3.6$ & $8.7 \pm 3.5$ \\
\hline $\begin{array}{l}\text { Number of high risk } \\
\text { prescriptions }\end{array}$ & $1018 \pm 770.4$ & $1021 \pm 773.3$ \\
\hline $\begin{array}{l}\text { Days' supply of high risk } \\
\text { prescriptions }\end{array}$ & $25.5 \pm 18.8$ & $24.9 \pm 18.4$ \\
\hline
\end{tabular}

$P P O$ Preferred provider organization, $H M O$ Health maintenance organization, POS Point-of-service, EPO Exclusive provider organization

${ }^{a}$ Data are reported as mean \pm standard deviation for: age, Charlson index, baseline concomitant medications, number of high risk prescriptions, and days' supply of high risk prescriptions

b Traumatic SCI defined as ICD-9 code of 806.xx or 952.xx. Nontraumatic SCI defined as ICD-9 code of $344.0 \mathrm{x}$ or $344.1 \mathrm{x}$ level-specific codes, the most common SCI injury level was Lumbar/Sacral ( $n=347,12.5 \%$ of total SCI cohort), followed by T7-T12 ( $n=216,7.8 \%$ of total SCI cohort). A majority of persons in the SCI cohort had non-traumatic mechanisms of injury $(63.3 \%)$.

Descriptive examination of the outcome variable, number of ADEs, revealed that 876 participants with SCI (31.5\%) and 676 controls $(24.3 \%)$ had at least one ADE. The most common ADEs were rash, dermatitis, and drug psychosis. Among persons with SCI, $24.9 \%$ had at least one rash, $4.0 \%$ had dermatitis, and $0.5 \%$ had drug psychosis. Among the control cohort, $16.3 \%$ had at least one rash, $5.1 \%$ had dermatitis, and $0.8 \%$ had drug psychosis.

Results of the conditional logistic regression estimating the occurrence of any ADE during the post-baseline period are shown in Table 2. Findings revealed that individuals with $\mathrm{SCI}(\mathrm{OR}=1.45, p<0.0001)$ and females $(\mathrm{OR}=1.38$, $p<0.0001$ ) were significantly more likely to have an ADE. Additionally, those with higher numbers of concomitant prescriptions $(\mathrm{OR}=1.05, p<0.002)$ during the baseline period were slightly more likely to experience an ADE. There was no significant relationship observed between risk of $\mathrm{ADE}$ and age in decades, hospital admission during baseline, or Charlson Index.

Among those with ADEs, the distribution of baseline characteristics was similar between persons with and without SCI, as evidenced by standardized mean differences $<0.25$ for all variables used in propensity score matching. As a result, we did not conduct a second round of propensity score matching prior to analyses comparing frequency of ADEs between cohorts for those with at least one ADE $(n=1552)$. Results of a Poisson regression (Table 3) estimating total number of ADEs revealed that, relative to the control cohort, the SCI cohort had significantly fewer ADEs after controlling for other variables in the model (IRR $=0.91, p<0.0001)$. Findings also revealed that individuals with a hospital admission (IRR $=1.36, \quad p<0.0001)$ and greater number of concomitant medications during baseline (IRR $=1.02$, $p<0.0001)$ had significantly higher rates of ADEs. For every 10-year increase in age, individuals tended to have significantly fewer ADEs, although this trend was not statistically significant $(\mathrm{IRR}=0.93, p=0.05)$. Female gender and Charlson Index were not significantly associated with a difference in the frequency of ADEs.

As patients with SCI may be more likely to undergo regular outpatient follow-up care, we conducted sensitivity analyses to control for the number of outpatient visits in the 12-month post-baseline period. Results of the logistic and Poisson regression models did not substantially change after controlling for number of outpatient visits. 
Fig. 2 Scatter plot illustrating the standardized mean differences for continuous variables between cohorts before and after propensity score matching. Dashed lines at \pm 0.25 represent the threshold of acceptable similarity between cohorts. HR: high-risk, Obs: observations.

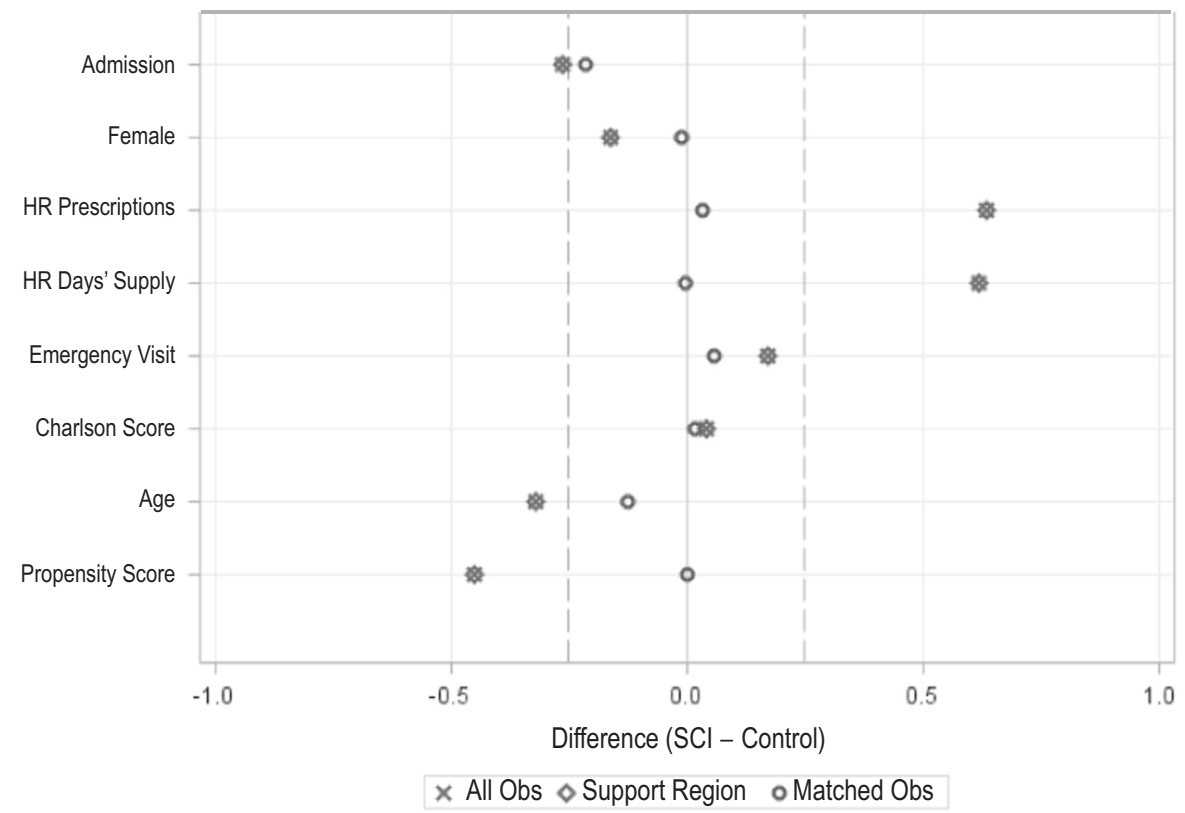

Table 2 Conditional logistic regression estimating likelihood of having an adverse drug event

\begin{tabular}{lllll}
\hline Variable & $\begin{array}{l}\text { Odds } \\
\text { ratio }\end{array}$ & $95 \%$ CI & $\chi^{2}$ & $P$ \\
\hline SCI & 1.45 & $1.28-1.64$ & 33.43 & $<0.0001$ \\
Female gender & 1.38 & $1.15-1.66$ & 11.85 & $<0.001$ \\
Charlson index & 1.14 & $1.00-1.30$ & 3.98 & 0.05 \\
$\begin{array}{l}\text { Hospital admission during } \\
\text { baseline }\end{array}$ & 1.23 & $0.97-1.57$ & 2.89 & 0.09 \\
$\begin{array}{l}\text { Decade age } \\
\begin{array}{l}\text { Mean concomitant } \\
\text { prescriptions }\end{array}\end{array}$ & 1.01 & $0.90-1.13$ & 0.03 & 0.86 \\
\hline
\end{tabular}

${ }^{\mathrm{a}}$ Mean concomitant prescriptions $=$ average number of concomitant prescriptions per month during the baseline period

Table 3 Poisson regression estimating total number of adverse drug events

\begin{tabular}{llll}
\hline Variable & IRR & $\mathbf{9 5 \%}$ CI & $\boldsymbol{P}$ \\
\hline SCI & 0.91 & $0.78-1.06$ & $<0.0001$ \\
Female gender & 0.97 & $0.83-1.14$ & 0.20 \\
Charlson index & 1.07 & $0.98-1.16$ & 0.72 \\
Hospital admission during baseline & 1.36 & $1.13-1.64$ & 0.04 \\
Decade age $_{\text {Mean concomitant prescriptions }^{\mathrm{a}}}$ & 0.93 & $0.86-1.01$ & 0.05 \\
\hline
\end{tabular}

${ }^{a}$ Mean concomitant prescriptions $=$ average number of concomitant prescriptions per month during the baseline period. IRR $=$ Incidence rate ratio

\section{Discussion}

The purpose of this study was to compare rates of ADEs among persons with polypharmacy with and without SCI.
In accordance with our hypothesis and previous literature [13], findings revealed that persons with SCI were significantly more likely than matched controls to have an ADE. These findings highlight the importance of considering patterns of polypharmacy-related ADEs among those with SCI, as they are different from patterns in a control population matched on number of concomitant medications, age, gender, geographic region, insurance plan type, emergency room visits, and inpatient hospital admissions. Findings also revealed that among participants with ADEs, persons with SCI had significantly fewer ADEs over time than the matched cohort after controlling for a number of potentially confounding factors. This finding is contrary to our hypothesis and previous literature [13] and suggests that the other factors included in the model may have accounted for previously observed findings.

Results of this study suggest that while persons with SCI and polypharmacy are at a greater risk for experiencing an $\mathrm{ADE}$, their medical care after an ADE may be better managed than that of a matched control population. One possible explanation for this may be increased implementation of the Chronic Care Model, which is a guide to improve quality of chronic condition management within primary care settings [23]. Persons with SCI report receiving care that more strongly aligns with the Chronic Care Model when compared to reports from a broader population of persons with chronic health conditions [24, 25], which may partly explain why persons with SCI have fewer ADEs over time than matched controls.

Similarly, results revealed that female gender was predictive of incidence of any ADE, but also predictive of fewer ADEs over time. The finding that females are more likely to experience ADEs than males is consistent with 
existing literature [26-29]. Various explanations for this gender difference have been posited, including the use of different drugs and gender-based physiological differences [30]. While females in the present study were more likely to have an initial ADE, they had significantly fewer ADEs over time, which may suggest that, similar to the SCI population, care post-ADE may be better managed for females.

After controlling for age, gender, medical comorbidity indices, and other factors, the current findings raise significant concerns about the incidence of polypharmacyrelated ADEs in persons with SCI. Therefore, the known risks of polypharmacy in the general population and older adults may be magnified after SCI, which has significant implications for clinical practice guidelines. Clinicians working with patients with SCI should carefully monitor polypharmacy and consider potential benefits and risk of harm when prescribing medications. Additionally, these findings suggest there may be a need for development of practice guidelines that facilitate proactive identification of persons with SCI at the highest risk of ADE so steps may be taken to mitigate risk; this approach is in contrast to current practice trends, which appear to take a reactive approach after an ADE has occurred.

\section{Strengths and limitations}

There are some important methodologic considerations for interpreting our findings. First, we cannot be certain about the degree of prescription adherance for individuals in this study. Diversion of prescription medications may be a problem among those with SCI, as they are an economically impoverished group [31]. Thus, findings may be interpreted as patterns of medication prescription, rather than medication use.

Second, the current findings reflect patterns of prescription medication use among persons who have private insurance. The patterns among those who are uninsured and those who have Medicaid or Medicare may be different. Additionally, due to the use of MarketScan data, information about other variables (e.g., ethnicity, socioeconomic status) that may account for observed findings is unknown. The effect of such variables should be considered in future prospective studies.

Third, the data did not include SCI-level specific diagnostic codes for $67.3 \%$ of persons in the SCI cohort, which limits our understanding of the demographics of the current sample and does not support more detailed subgroup analyses. Similarly, we do not have data on neurologic completeness of injury. Those with neurologically incomplete injuries, even with higher neurologic levels, are more likely to be ambulatory. Due to the absence of level-specific diagnostic codes for a majority of the SCI cohort and information about neurologic completeness, it is unclear whether results may best generalize to persons with more or less severe SCI. However, considering that among participants for whom neurologic level-specific codes were available, the greatest portion had either lumbar-sacral (12.5\% of total SCI cohort) or lower thoracic $(7.8 \%$ of total SCI cohort) injuries, the sample may be skewed towards less severe SCI.

The present study also has a number of methodologic strengths. First, findings offer valuable insights regarding prescription patterns and ADEs in a large, national, population-based sample of persons with SCI. By capitalizing on population-based insurance claims data spanning two years, trends may be revealed that would not be detected in smaller samples or with shorter follow-up. Second, the use of propensity score matching increases the robustness of our findings. This methodology ensures a greater chance that the observed differences were due to SCI diagnostic status rather than baseline demographic differences. Third, the present study included participants with traumatic or non-traumatic mechanisms of SCI. While is important to acknowledge that demographics and functional outcomes of persons with traumatic vs. non-traumatic SCI may be different $[32,33]$, we sought to compare trends in polypharmacy and ADEs among individuals with any SCI to a matched control population. Inclusion of persons with traumatic or non-traumatic mechanisms of injury allows findings of this study to build upon other recent works [13] with enhanced generalizability to the broader population of people with SCI.

\section{Future directions}

To build further upon these findings, future research should focus on developing effective strategies for detecting elevated risk of ADEs among persons with polypharmacy and SCI. As a result, clinicians may be better equipped to prevent ADEs in this population. Additionally, future studies should examine the relationship between polypharmacy, ADEs, and a variety of outcomes such as functional independence, quality of life, satisfaction with care, and mortality. Such work would add to current findings, which reveal negative health consequences of polypharmacy in SCI, by facilitating an understanding of the broader impact of polypharmacy in this population.

\section{Conclusion}

Findings of this study indicate that, among individuals with polypharmacy, persons with SCI demonstrated significantly different ADE outcomes than matched controls. Specifically, persons with SCI were more likely to experience an 
ADE than matched controls. Among persons with ADEs, individuals with SCI experienced fewer ADEs over time than matched controls.

Acknowledgements This work was supported by the South Carolina Spinal Cord Injury Research Fund, grant numbers \#2016 PD-01 and \#09-001. This study was partially supported by the South Carolina Clinical and Translational Research (SCTR) Institute, with an academic home at the Medical University of South Carolina, through NIH-NCATS Grant Number UL1 TR001450. Data analytic support for the study was provided through support for the CEDAR core funded by the MUSC Office of the Provost.

\section{Compliance with ethical standards}

Conflict of interest The authors declare that thay have no conflict of interest.

\section{References}

1. Guthrie B, Makubate B, Hernandez-Santiago V, Dreischulte T. The rising tide of polypharmacy and drug-drug interactions: population database analysis 1995-2010. BMC Med. 2015;13:74.

2. Fried TR, O'Leary J, Towle V, Goldstein MK, Trentalange M, Martin DK. Health outcomes associated with polypharmacy in community-dwelling older adults: a systematic review. J Am Geriatr Soc. 2014;62:2261-72.

3. Cashion W, McClellan W, Howard G, Goyal A, Kleinbaum D, Goodman M, et al. Geographic region and racial variations in polypharmacy in the United States. Ann Epidemiol. 2015;25:433-8. e1

4. Veehof L, Stewart R, Haaijer-Ruskamp F, Jong BM. The development of polypharmacy. A longitudinal study. Fam Pract. 2000;17:261-7.

5. Scott IA, Hilmer SN, Reeve E, Potter K, Le Couteur D, Rigby D, et al. Reducing inappropriate polypharmacy: the process of deprescribing. JAMA Int Med. 2015;175:827-34.

6. Scott IA, Anderson K, Freeman CR, Stowasser DA. First do no harm: a real need to deprescribe in older patients. Med J Aust. 2014;201:390-2.

7. Jensen EK, Biering-Sorensen F. Medication before and after a spinal cord lesion. Spinal Cord. 2014;52:358-63.

8. Shnek ZM, Foley FW, LaRocca NG, Gordon WA, DeLuca J, Schwartzman HG, et al. Helplessness, self-efficacy, cognitive distortions, and depression in multiple sclerosis and spinal cord injury. Ann Behav Med. 1997;19:287-94.

9. Lee AK, Miller WC, Townson AF, Anton HA. Medication use is associated with fatigue in a sample of community-living individuals who have a spinal cord injury: A chart review. Spinal Cord. 2010;48:429-33.

10. Saunders LL, Krause JS. Personality and behavioral predictors of pressure ulcer history. Top Spinal Cord Inj Rehabil. 2010;16:61-71.

11. Hope ME, Kailis SG. Medication usage in a spinal cord injured population. Spinal Cord. 1998;36:161-5.

12. Hwang M, Zebracki K, Vogel LC. Medication profile and polypharmacy in adults with pediatric-onset spinal cord injury. Spinal Cord. 2015;53:673-8.

13. Kitzman P, Cecil D, Kolpek JH. The risks of polypharmacy following spinal cord injury. J Spinal Cord Med. 2017;40: 147-53.
14. Flaherty JH, Perry HM 3rd, Lynchard GS, Morley JE. Polypharmacy and hospitalization among older home care patients. J Gerontol A Biol Sci Med Sci. 2000;55:M554-9.

15. Jyrkka J, Enlund H, Korhonen MJ, Sulkava R, Hartikainen S. Polypharmacy status as an indicator of mortality in an elderly population. Drugs Aging. 2009;26:1039-48.

16. Rosenbaum PR, Rubin DB. The central role of the propensity score in observational studies for causal effects. Biometrika. 1983;70:41-55.

17. Charlson ME, Pompei P, Ales KL, MacKenzie CR. A new method of classifying prognostic comorbidity in longitudinal studies: development and validation. $J$ Chron Dis. 1987; $40: 373-83$.

18. Elixhauser A, Steiner C, Harris DR, Coffey RM. Comorbidity measures for use with administrative data. Med Care. 1998;36:8-27.

19. Coca-Perraillon M. Local and global optimal propensity score matching. SAS Glob Forum. 2007;185:1-9.

20. Stuart EA. Matching methods for causal inference: a review and a look forward. Stat Sci. 2010;25:1-21.

21. Tuinen MV, Elder S, Link C, Li S, Song JH, Pritchett T. Surveillance of surgery-related adverse events in Missouri using ICD9-CM codes. In: Henriksen K, Battles JB, Marks ES, Lewin DI, editors. Advances in patient safety: From research to implementation (Volume 1: Research Findings). Advances in patient safety. Agency for Healthcare Research and Quality (US), Rockville, MD; 2005.

22. SAS Institute Inc. SAS for Windows. 9.4 edition. Cary, NC: SAS Institute; 2015.

23. Bodenheimer $\mathrm{T}$, Wagner $\mathrm{EH}$, Grumbach K. Improving primary care for patients with chronic illness. JAMA. 2002;288: 1775-9.

24. Heinemann AW, LaVela SL, Etingen B, Miskovic A, Locatelli SM, Neumann HD, et al. Perceptions of person-centered care following spinal cord injury. Arch Phys Med Rehabil. 2016;97:1338-44.

25. Glasgow RE, Wagner EH, Schaefer J, Mahoney LD, Reid RJ, Greene SM. Development and validation of the Patient Assessment of Chronic Illness Care (PACIC). Med Care. 2005;43:436-44.

26. Chan M, Nicklason F, Vial JH. Adverse drug events as a cause of hospital admission in the elderly. Intern Med. 2001;31:199-205.

27. Evans RS, Lloyd JF, Stoddard GJ, Nebeker JR, Samore MH. Risk factors for adverse drug events: a 10-year analysis. Ann Pharmacother. 2005;39:1161-8.

28. Chen YC, Fan JS, Chen MH, Hsu TF, Huang HH, Cheng KW, et al. Risk factors associated with adverse drug events among older adults in emergency department. Eur $\mathbf{J}$ Intern Med. 2014;25:49-55.

29. Fushiki Y, Kinoshita K, Tokuda Y. Polypharmacy and adverse drug events leading to acute care hospitalization in Japanese elderly. J Gen Fam Med. 2014;15:110-6.

30. Rademaker M. Do women have more adverse drug reactions? Am J Clin Dermatol. 2001;2:349-51.

31. Krause JS, Dismuke CE, Acuna J, Sligh-Conway C, Walker E, Washington K, et al. Race-ethnicity and poverty after spinal cord injury. Spinal Cord. 2014;52:133-8.

32. Guilcher SJ, Munce SE, Couris CM, Fung K, Craven BC, Verrier $\mathrm{M}$, et al. Health care utilization in non-traumatic and traumatic spinal cord injury: a population-based study. Spinal Cord. 2010;48:45-50.

33. New PW, Simmonds F, Stevermuer T. A population-based study comparing traumatic spinal cord injury and non-traumatic spinal cord injury using a national rehabilitation database. Spinal Cord. 2011;49:397-403. 